\title{
In Vitro Selection of Fab Fragments by mRNA Display and Gene-Linking Emulsion PCR
}

\author{
Takeshi Sumida, Hiroshi Yanagawa, and Nobuhide Doi \\ Department of Biosciences and Informatics, Keio University, 3-14-1 Hiyoshi, Kohoku-ku, Yokohama 223-8522, Japan \\ Correspondence should be addressed to Nobuhide Doi, doi@bio.keio.ac.jp
}

Received 29 May 2012; Revised 1 August 2012; Accepted 1 August 2012

Academic Editor: Hiroshi Murakami

Copyright ( $) 2012$ Takeshi Sumida et al. This is an open access article distributed under the Creative Commons Attribution License, which permits unrestricted use, distribution, and reproduction in any medium, provided the original work is properly cited.

\begin{abstract}
In vitro selection by display methods has been an effective tool for engineering recombinant antibodies. mRNA display based on a cell-free translation system has the advantages of larger library sizes and quicker selection procedures compared with cell-based display methods such as phage display. However, mRNA display has been limited to select single-chain polypeptides such as scFvs due to its characteristic of linking a nascent polypeptide with its encoding mRNA on the ribosome. Here we demonstrated a new way of selecting heterodimeric Fab fragments by using mRNA display combined with emulsion PCR. We designed a pair of complementary $5^{\prime}$ UTR sequences that can link the Fab heavy and light chain genes together by overlap-extension PCR in waterin-oil emulsions. We confirmed that two mRNA-displayed polypeptides for heavy and light chain of a model Fab fragment were associated into the active form and that a specific Fab fragment gene was enriched over 100-fold per round of a model affinity selection followed by the gene-linking emulsion PCR. We further performed directed evolution of Fab fragments with higher binding activity from a randomized Fab fragment library.
\end{abstract}

\section{Introduction}

In vitro selection by display methods has been an effective tool in the field of protein engineering and especially has been used to engineer recombinant antibodies for various biological applications [1]. Phage display has been widely used in the industry due to its feasibility to select Fab fragments [2]. The Fab fragment of an immunoglobulin is a heterodimer of the $\mathrm{N}$-terminal half of a heavy $(\mathrm{H})$ chain and a complete light (L) chain. Because the Fab is more native-like than the single-chain $\mathrm{Fv}(\mathrm{scFv})$, which is the other commonly used recombinant antibody format for in vitro selection, the Fab fragment format makes it able to select more practical antibodies [3]. Other than phage display, cell-free translation-based methods such as ribosome display [4] and mRNA display [5] are being used for in vitro selection of antibodies due to its advantage of permitting speedier selection from larger size libraries than cell-based methods. However, these cell-free translation-based methods are limited to select scFvs due to its characteristic of linking a nascent polypeptide with its encoding mRNA on the ribosome.

To overcome this limit, we have recently developed a bicistronic DNA display to select Fab fragments in a cellfree translation system [6]. Bicistronic DNA display relies on in vitro compartmentalization in water-in-oil emulsions [7], and the man-made cell-like compartments make it possible to display oligomeric proteins in a cell-free translation system. Although bicistronic DNA display has made it possible to select Fab fragments in a cell-free translation system, it has some disadvantages compared with mRNA display. First, the initial library size of bicistronic DNA display is three orders of magnitude less than that of mRNA display. Second, the linkage between the DNA and protein is a streptavidin-biotin complex, making it less stable compared with the covalent bond in mRNA display.

In this study we combined emulsion PCR [8-11] with mRNA display in order to be able to select Fab fragments by mRNA display. Since mRNA display is capable of selecting candidates from a more diverse library and designing a more 


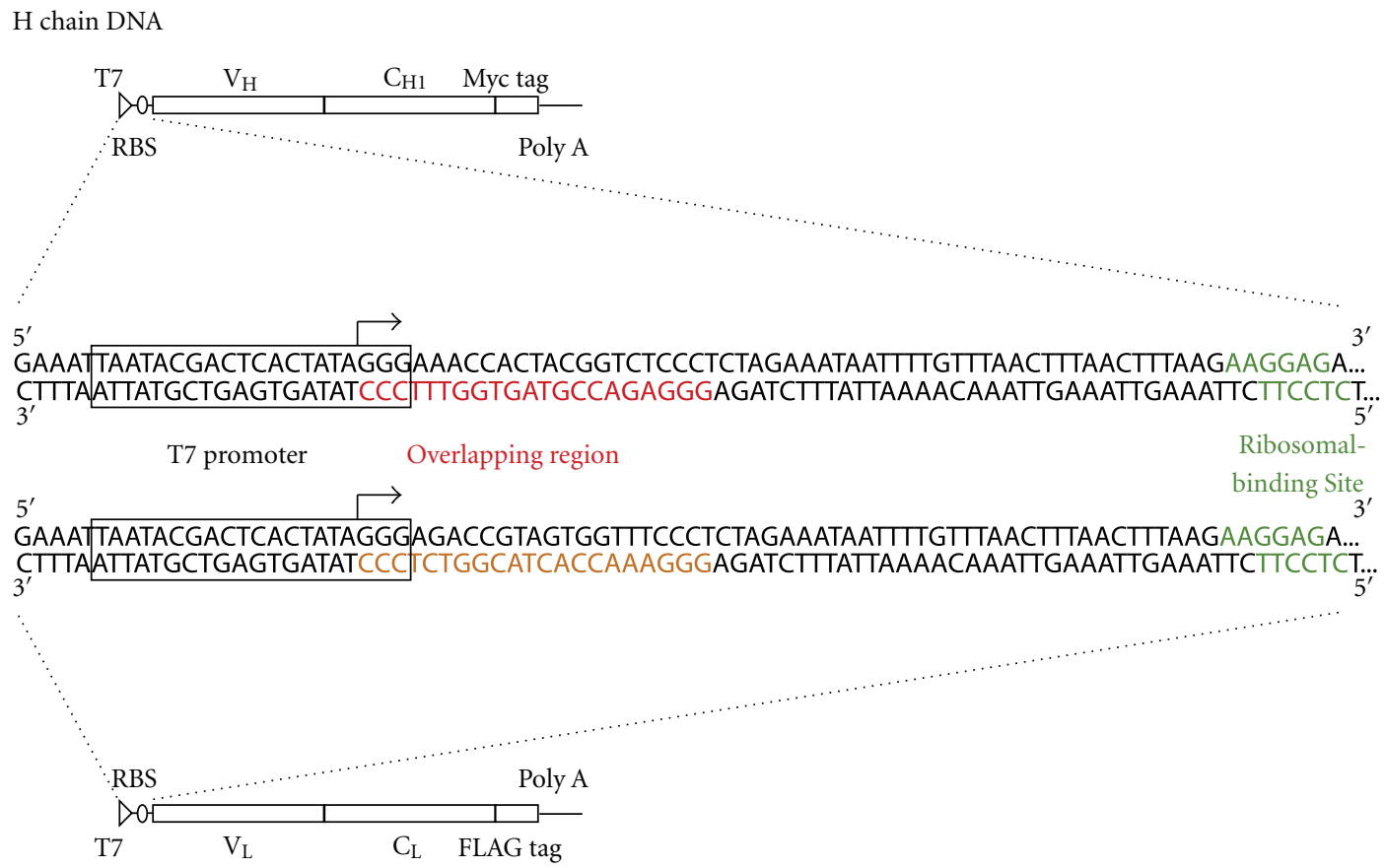

L chain DNA

(a)

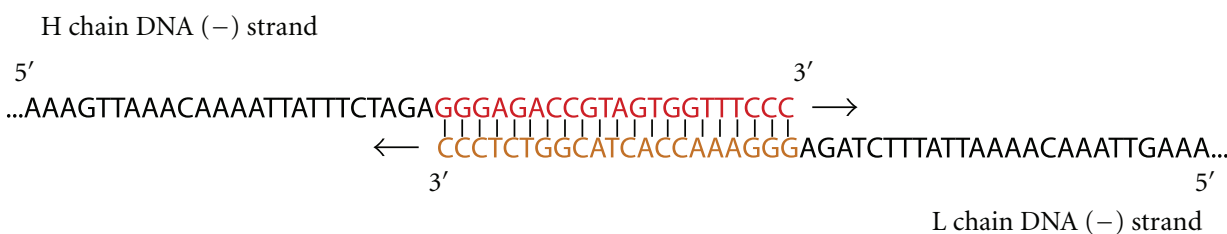

(b)

FIgure 1: The DNA construct of the Fab fragments for mRNA display. (a) From the $5^{\prime}$ end it consists of a T7 promoter (T7), ribosomalbinding site (RBS), variable region and constant region of the $\mathrm{H}$ chain or $\mathrm{L}$ chain, epitope tag, and a poly $\mathrm{A}$ tail. The $\mathrm{H}$ chain epitope tag is a Myc tag and the L chain is a FLAG tag. The poly A tail is for purification of mRNA-displayed molecules by biotin-oligo dT in combination with streptavidin beads. (Middle) Details of the linkable 5' UTR. Between the T7 promoter (boxed) and RBS (green), 21 bases from the beginning of T7 promoter transcription start point (arrow), in other words +1 to +21 of the $\mathrm{H}$ chain DNA (red) and L chain DNA (orange) are designed so that they overlap with each other during overlap-extension PCR. (b) The reverse-transcribed DNA strands overlap at the overlapping region (red) to form an $\mathrm{H}$ chain gene and L-chain-gene-linked DNA.

flexible selection strategy compared with bicistronic DNA display, this new method would provide a new option for selecting Fab fragments in a cell-free translation system.

\section{Results and Discussion}

2.1. Strategy. A Fab fragment consists of an $\mathrm{H}$ chain and an L chain, and by applying mRNA display, an mRNA-displayed $\mathrm{H}$ chain and an mRNA-displayed $\mathrm{L}$ chain can each be made. If these two mRNA-displayed molecules dimerize, they will form an mRNA-displayed Fab fragment. However, in this case, the correspondence of the selected $\mathrm{H}$ and $\mathrm{L}$ chains cannot be determined because the two genes are different RNA molecules and will be amplified separately after affinity selection. Applying overlap-extension PCR in water-in-oil emulsion from a single Fab molecule and linking these two genes together to amplify them at once will overcome this problem. Thus, we have designed a pair of complementary $5^{\prime}$ UTR sequences that can be linked together by overlapextension PCR (Figure 1). The whole DNA construct for this strategy consists of a linkable $5^{\prime}$ UTR with a T7 promoter and ribosomal binding site; an ORF with the variable region, constant region, and an affinity tag, and at the $3^{\prime}$ end there are 25 adenines for mRNA-based purification by oligo-dT resin.

The scheme for in vitro selection of Fab fragments using mRNA display and emulsion PCR is shown in Figure 2. Firstly, mRNA-displayed $\mathrm{H}$ and $\mathrm{L}$ chains are separately prepared by in vitro translation of puromycin-ligated mRNA templates. Both the $\mathrm{H}$ chain and $\mathrm{L}$ chain are subsequently purified by oligo-dT resin in order to remove all free proteins that could not form an mRNA-displayed molecule, avoiding a Fab fragment existing with only one chain being 

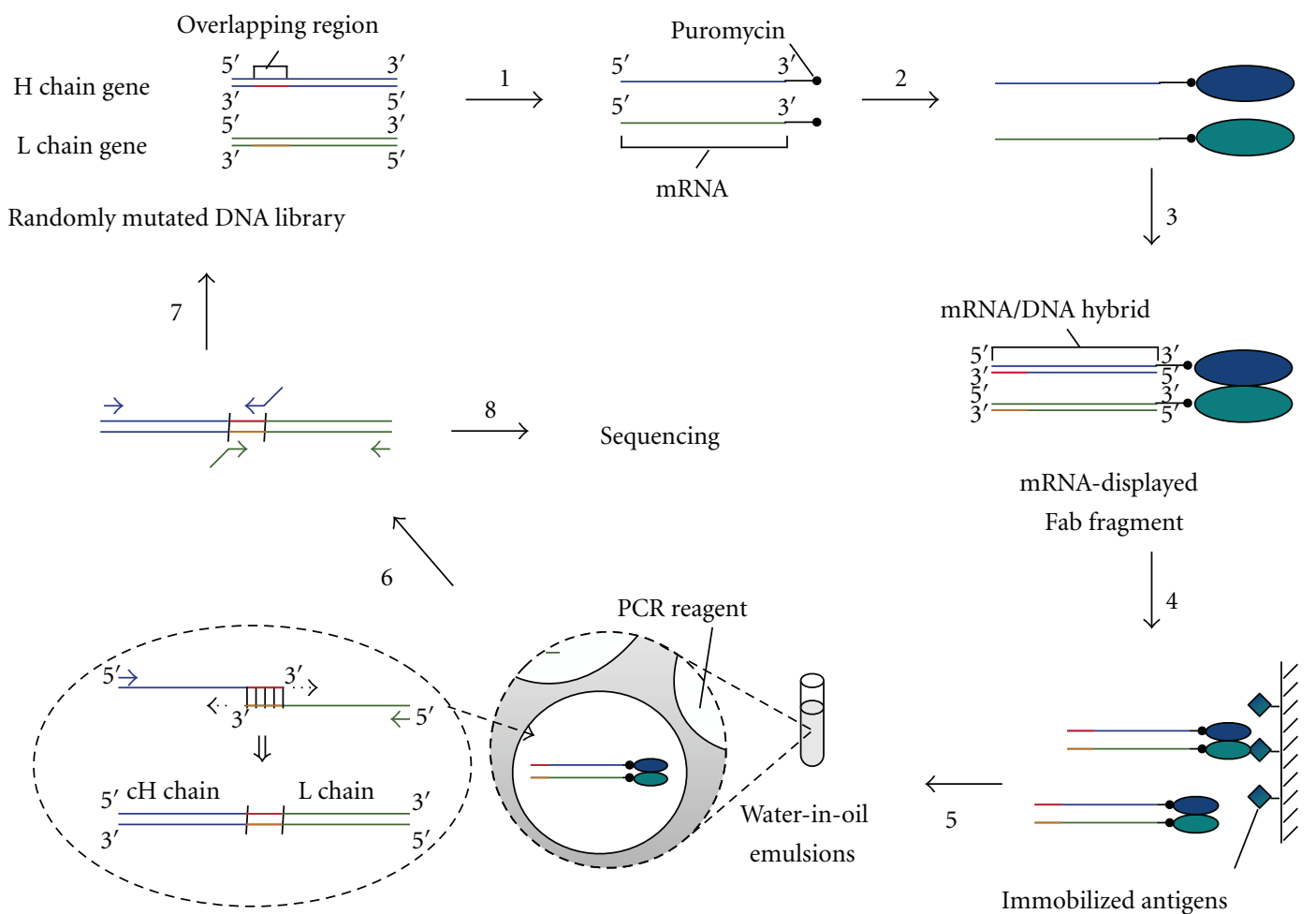

Linkage of selected $\mathrm{H}$ and $\mathrm{L}$ chain by emulsion PCR

FIGURE 2: Scheme of in vitro selection of Fab fragments using mRNA display and emulsion PCR. Step 1: A randomly mutated DNA library of an $\mathrm{H}$ chain gene and an L chain gene is separately prepared. Each DNA library is transcribed and puromycin is ligated to the $3^{\prime}$ end to make an mRNA template. Step 2: The mRNA template is translated to form an mRNA-displayed molecule. Step 3: The molecules are purified and subsequently reverse is transcribed to make the mRNA portion a DNA hybrid. The $\mathrm{H}$ and $\mathrm{L}$ chain molecules are combined to form an mRNA-displayed Fab fragment. Step 4: The mRNA-displayed Fab fragments are subjected to in vitro antigen selection. Step 5: The selected mRNA-displayed Fab fragments are recovered and mixed with PCR reagents. The mixture is emulsified and the corresponding H and L chain genes are linked together by overlap extension PCR. Step 6: The linked DNA is amplified by PCR again with different primers to regenerate the $\mathrm{H}$ and $\mathrm{L}$ chain genes. Step 7: The regenerated genes are reamplified by DNA shuffling to make the templates for the next round of selection. Step 8: After a suitable number of rounds of selection, the selected DNA is cloned and sequenced to identify the selected Fab fragments.

mRNA-displayed. After a reverse transcription step in order to make the mRNA portion of the mRNA-displayed molecule an mRNA/DNA hybrid, the $\mathrm{H}$ and $\mathrm{L}$ chains are mixed together to form mRNA-displayed Fab fragments. These mRNA-displayed Fab fragments are selected by the target antigen and then eluted under conditions that the oligomeric structure is maintained. The eluted mRNA-displayed Fab fragments are subjected to emulsion PCR and a single mRNA-displayed Fab fragment is trapped inside a single micelle where the $\mathrm{H}$ and $\mathrm{L}$ chain genes are linked together by overlap-extension PCR. The linked DNA is either amplified by PCR to regenerate the $\mathrm{H}$ and $\mathrm{L}$ chain genes for further selection or sequenced to identify the selected Fab fragments.

2.2. Proof-of-Principle Experiments. In order for the scheme depicted in Figure 2 to work, we confirmed the following three points: (i) the $\mathrm{H}$ and $\mathrm{L}$ chain genes overlap properly by emulsion PCR; (ii) an mRNA-displayed $\mathrm{H}$ chain and an mRNA-displayed L chain form a Fab fragment with binding activity; (iii) genes do not crossover during emulsion PCR and the corresponding $\mathrm{H}$ chain and $\mathrm{L}$ chain genes are properly linked together.

Figure 3(a) shows that when there are only reverse primers for the $\mathrm{H}$ and $\mathrm{L}$ chains, the DNA amplifies only when both the $\mathrm{H}$ and $\mathrm{L}$ chain genes exist and does not when there is only one of the genes. This proves that the linkable $5^{\prime}$ UTR is properly designed to overlap and only Fab fragments with both mRNA-displayed $\mathrm{H}$ and $\mathrm{L}$ chains can be specifically amplified. Therefore, unwanted gene amplification of unspecific $\mathrm{H}$ chain or $\mathrm{L}$ chain binders that does not form Fab fragments can be removed, exhibiting the advantage of this overlapping method compared to regular PCR amplification.

Next, to show that an mRNA-displayed $\mathrm{H}$ chain and mRNA-displayed $\mathrm{L}$ chain form a Fab fragment with binding activity, mRNA-displayed anti-fluorescein Fab and antip53 Fab fragments were used in a model affinity selection experiment. Confirmed by pull-down assays and western blotting, these antibodies do not bind unless they form a Fab fragment (data not shown). Equal molar amounts of mRNA-displayed anti-fluorescein Fab and anti-p53 Fab fragments were subjected to in vitro selection against antigen 

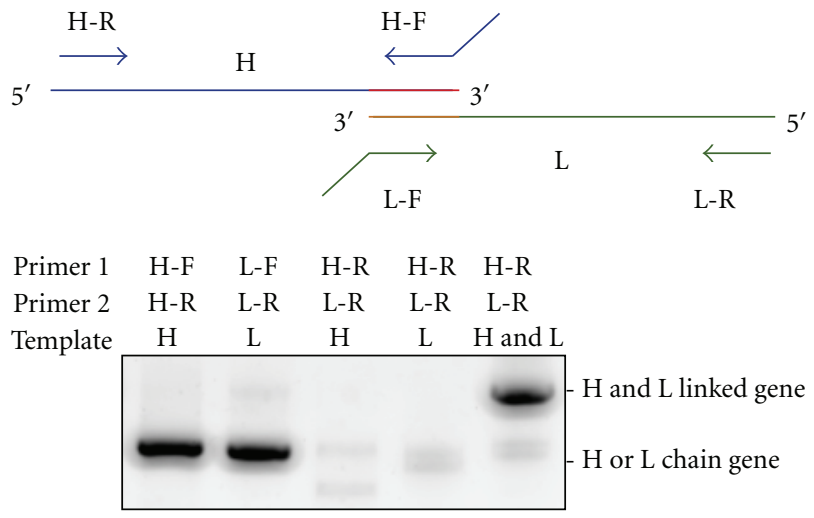

(a)

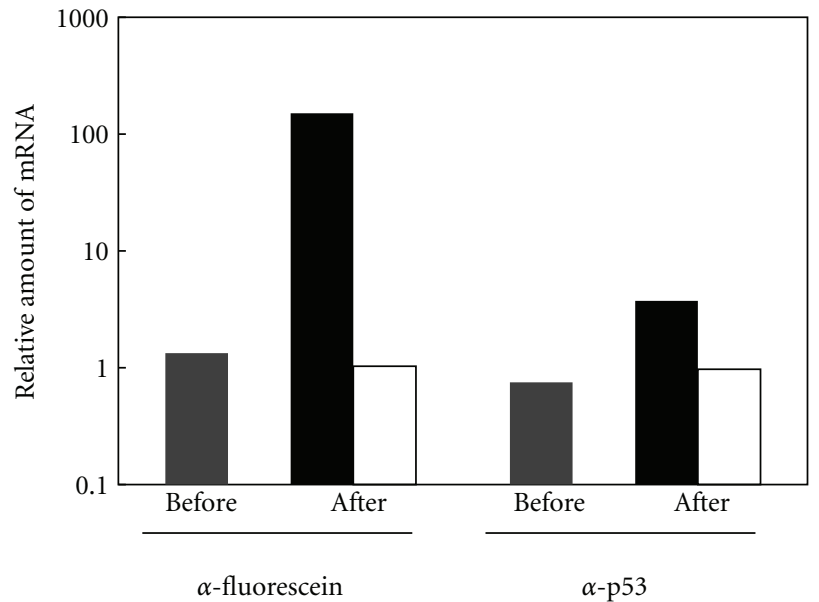

(b)

\begin{tabular}{|c|c|c|c|}
\cline { 3 - 4 } \multicolumn{2}{c|}{} & \multicolumn{2}{c|}{ L chain } \\
\cline { 3 - 4 } \multicolumn{2}{c|}{} & Fluorescein & $\mathrm{p} 53$ \\
\hline \multirow{3}{*}{ H chain } & Fluorescein & 11 & 0 \\
\cline { 2 - 4 } & $\mathrm{p} 53$ & 0 & 2 \\
\hline
\end{tabular}

(c)

Figure 3: Model experiments of in vitro selection of Fab fragments using mRNA display and emulsion PCR. (a) Confirmation of the H and $\mathrm{L}$ chain gene linking by PCR. The H chain or/and L chain gene of anti-fluorescein Fab fragments were amplified by PCR with forward and reverse primers of the corresponding gene or only reverse primers of both genes and analyzed on a $1.5 \%$ agarose gel. Primers $\mathrm{H}-\mathrm{F}$, $\mathrm{H}-\mathrm{R}$, L-F and L-R are UnivOL1-F, Myc-R, UnivOL2-F, and FLAG-R, respectively (Table 1). (b) Antigen-specific enrichment of mRNA-displayed Fab fragments. A single round of affinity selection was carried out for antigen-immobilized and nonimmobilized beads from a mixture of equally amounted anti-p53 and anti-fluorescein Fab fragment genes. The amount of each gene before and after selection was quantified by quantitative PCR. The amount of the positive control gene divided by that of the negative control gene is plotted for both before selection (gray) and after selection by antigen-immobilized bead (black) and nonimmobilized beads (white). (c) Confirmation of $\mathrm{H}$ and $\mathrm{L}$ chain gene linking by cloning and sequencing after affinity selection. mRNA-displayed anti-fluorescein Fab fragments and mRNA-displayed anti-p53 Fab fragments were mixed in a ratio of $1: 50$ and then used for affinity selection against fluorescein-immobilized beads. After selection, a total of 13 clones were sequenced, and the correspondence of the $\mathrm{H}$ and $\mathrm{L}$ chains was confirmed.

p53 or fluorescein, and the amounts of the molecules before and after selection were quantified by quantitative PCR. As expected, each of the Fab fragment genes was enriched when they were selected against their antigens (Figure 3(b)). The anti-fluorescein Fab fragment gene showed an approximate 110-fold enrichment and the anti-p53 Fab fragment gene showed an approximate 5-fold enrichment. The difference in the enrichment efficiency is probably due to the difference in the dissociation constant; the anti-p53 Fab fragment has a weaker affinity towards its antigen. On the other hand, both antibodies did not show any enrichment when their antigens were not present during in vitro selection, showing that both mRNA-displayed Fab fragments bind to their antigens with specificity.

Finally, to confirm that genes do not crossover during emulsion PCR and the corresponding $\mathrm{H}$ chain and $\mathrm{L}$ chain genes are properly linked together, the DNA after affinity selection was cloned and confirmed by capillary DNA sequencing. The mRNA-displayed anti-fluorescein Fab fragment and the mRNA-displayed anti-p53 Fab fragment were mixed in a ratio of $1: 50$ and then used for affinity selection against fluorescein-immobilized beads. Out of the 13 sequenced DNA clones after selection, 11 clones were $\mathrm{H}$ and $\mathrm{L}$ chain-linked anti-fluorescein and 2 clones were $\mathrm{H}$ and $\mathrm{L}$ chain-linked anti-p53 $(P<0.001)$, resulting in a conclusion that the corresponding $\mathrm{H}$ chain and $\mathrm{L}$ chain genes are properly linked together and the genes do not crossover during emulsion PCR (Figure 3(c)).

\subsection{Affinity Selection from a Randomized Fab Fragment} Library. Finally, we applied the mRNA display and emulsion PCR procedure for selection from a randomized Fab fragment library. We constructed an anti-p53 Fab fragment library with random point mutations by error-prone PCR and DNA shuffling from the wild type. When a fraction of the library was analyzed by DNA sequencing, the $\mathrm{H}$ chain and $\mathrm{L}$ chain had an average of 2.9 base/gene and 3.1 base/gene mutation, respectively. From this library, 4 rounds of affinity selection were performed under the condition of gradually decreasing amounts of immobilized antigen (round 1, $400 \mathrm{nM}$; round 2, $40 \mathrm{nM}$; round 3, $4 \mathrm{nM}$; round $4,0.4 \mathrm{nM})$. After 4 rounds of selection, the total binding activity of in-vitro-translated products of the library at each round was analyzed by ELISA (Figure 4(a)). The binding activity gradually increased in successive rounds of selection, 


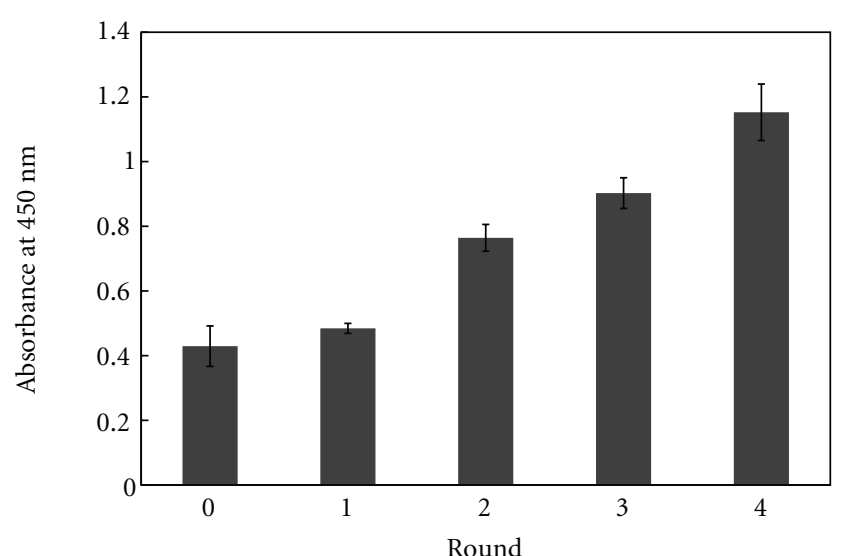

(a)

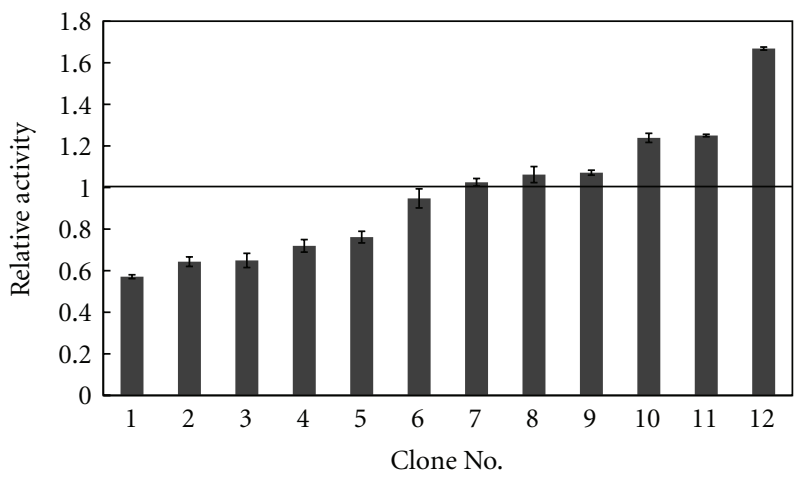

\begin{tabular}{|c|ccc|cc|}
\hline Clone no. & \multicolumn{3}{|c|}{ H chain } & \multicolumn{2}{c|}{ L chain } \\
\hline 1 & A98V & H164N & S187R & L11S & F121S \\
2 & K206R & & & Q17K & R46H \\
3 & A98V & S187R & & - & \\
4 & A98V & S187R & K206Q & - & \\
5 & A88T & S187R & & - & \\
6 & S161N & S187R & & - & \\
7 & S120P & S187R & K206R & - & \\
8 & S187R & & & - & \\
9 & K206R & & & - & \\
10 & K206R & & & V85A & \\
11 & S187R & & & K53R & \\
12 & - & & & S28N & P143L \\
\hline
\end{tabular}

(b)

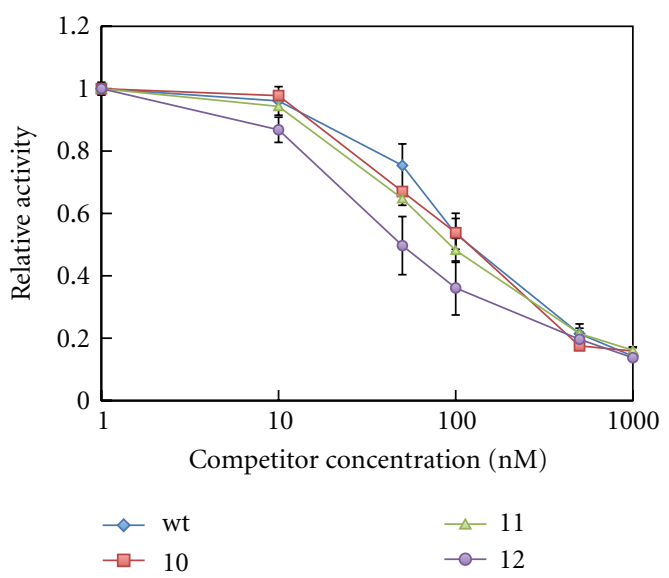

(d)

FIGURE 4: Affinity selection of randomly mutated anti-p53 Fab fragments. (a) Binding activity of the library after each round of selection. Random mutations were introduced to anti-p53 Fab fragments and 4 rounds of affinity selection were carried out under decreasing antigen concentration (round 1, $400 \mathrm{nM}$; round 2, $40 \mathrm{nM}$; round 3, $4 \mathrm{nM}$; round 4, $0.4 \mathrm{nM}$ ). After each round of selection, the fraction of the mutant Fab fragment library that binds to p53 was monitored by ELISA. Round 0 represents the randomly mutated initial library before selection. (b) Amino acid mutations of the selected variants. Bold type represents the mutations in the CDR and the minus (-) represents no amino acid mutations. (c) Binding activity of the selected variants after the 4th round of selection. The antigen binding activities of the selected variants were measured by ELISA. The absorbance at $450 \mathrm{~nm}$ of the wild type was used for normalization. (d) Competitive ELISA for estimating their affinities of variants with higher binding activity than the wild type. Competitive ELISA was preformed with $0-1000 \mathrm{nM}$ free p53 for clone number 10-12 and the wild type (wt). The signals were normalized to that of $0 \mathrm{nM}$ competitor concentration.

indicating that specific binders have been enriched in the library.

The library after the 4 th round of selection was cloned and sequenced (Figure 4(b)). The binding activities of arbitrarily chosen 12 clones were analyzed and approximately $60 \%$ of those clones had similar or higher binding activities than the wild type (Figure 4(c)). The clones that had similar binding activities as the wild type (numbers 6-9) had mutations only in the constant region and since the constant region does not influence the affinity of most antibodies $[3,6]$ it is likely that these are neutral mutations. Among these mutations, S187R and K206R were found in several other clones and, these may be fixed neutral mutations in an early round of selection by random genetic drift. The clones that had higher binding activity than the wild type (numbers 10-12) all had mutations in the variable region of the $\mathrm{L}$ chain, either in the CDR or close to the CDR. Since the CDR directly contacts with the antigen, mutations in and near the CDR affect the affinity of the antibody $[5,12-$ 14]. Analysis by competitive ELISA confirmed that all these clones specifically competed against free p53 (Figure 4(d)), and from the Scatchard plot, the $K_{d}$ of clone 10,11, 12 and wild type were estimated to be around $100 \mathrm{nM}, 90 \mathrm{nM}$, $60 \mathrm{nM}$, and $140 \mathrm{nM}$, respectively.

These results demonstrated that the combination of mRNA display and emulsion PCR was able to eliminate inactive Fab fragments from the randomized Fab fragment library and select Fab fragment candidates according to the designed procedure. However, the affinities of the selected mutants were not so high. Only mutations in the L chain had a positive influence on the affinity, and mutations in the $\mathrm{H}$ chain were either neutral (S187R, K206R) or slightly harmful 
(A98V). Mutants with A98V still have intermediate binding activity and the rather mild selection pressure of decreasing antigen concentration in this study may have let such a mutation survive through selection. Gradually lowering the antigen concentration has been demonstrated as a strategy for affinity maturation of some antibodies $[15,16]$ but introducing more stringent selection pressure such as offrate selection $[5,12-14]$ may have produced a better result. Further, the difference in the cell-free translation system may have affected the result as well. In this study, a reconstituted PURE E. coli translation system [17] was used for mRNA display of antibody fragments for the first time, but the efficiency of the mRNA being linked to the protein $(\sim 10 \%$ of the total mRNA library; data not shown) is lower than our previous study based on a wheat germ translation system [5]. Optimizing the cell-free translation system for our mRNA display system should produce better results as well.

2.4. Future Perspectives. A big advantage of our method in this study may be the large library size of mRNA display, which is the largest among all display methods, and the use of next generation sequencers would be able to pull out the full potential of this method. The newest Roche 454 Sequencer can sequence approximately $10^{6}$ reads of approximately $900 \mathrm{bp}$, long enough to cover both of the linked variable regions. Also, a recent study by phage display and deep sequencing has revealed that one round of selection is enough for identifying positive clones [18]. Although, $10^{6}$ reads are not enough to cover the whole selected Fab fragment library, combining it with a microfluidic chip for high enrichment efficiency per round of selection [19] may make it possible to obtain unique high affinity binders. Further possibilities may be considered when the specifications of the next generation sequencers improve even more. The speed of improvement for this technology is remarkable, and when it becomes possible to sequence the whole selected Fab fragment library (around $10^{8}-10^{9}$ molecules), it should allow selection of low affinity antibodies that would usually be lost in a typical selection of repetitious rounds, expanding the variety of potentially effective antibody candidates.

Other possible applications by our method described in this study would be proteome analyses, such as massively parallel detection of protein/protein interactions. Recently, Nirantar and Ghadessy demonstrated a way to identify various protein/protein interactions by library versus library two-hybrid screening using emulsion PCR [20]. A similar strategy can be carried out by our method by pulling-down in-vitro-translated mRNA-displayed protein complexes with an affinity tag and incorporating them into emulsions. Furthermore, the immense flexibility of our cell-free translationbased method would allow selection of not only protein/protein complexes, but also RNA/protein complexes. This concept can be completed by simply changing one side of the complex from an mRNA-displayed molecule to an ordinary RNA molecule, in other words merging SELEX [21], a common way for in vitro selection of RNA aptamers, with this mRNA display and emulsion PCR method. An example of RNA/protein complex selection would be selection of a ribonucleic peptide aptamer. In previous studies, it took two steps to make a high-affinity ribonucleic peptide aptamer against ATP, first step by SELEX [22] and second step by phage display [23]. Since our method can do selection against RNA and peptide at once, it may be possible to obtain a high-affinity binder in only one step. We have confirmed that emulsion RT-PCR can be carried out and incorporated into this method (data not shown), and selection of RNA/protein complexes by in vitro display methods shall be carried out in the near future.

In conclusion, other than in vitro selection of Fab fragments, our method in this study has the possibilities of being able to carry out proteomics applications and RNA/protein complex selection. This variety of possible applications shows the potential convenience of in vitro selection by mRNA display and gene-linking emulsion PCR.

\section{Materials and Methods}

3.1. DNA Construction. The oligonucleotide sequences used in this study are listed on Table 1 . The $\mathrm{H}$ chain and $\mathrm{L}$ chain genes of both anti-fluorescein Fab and anti-p53 Fab fragments were constructed by PCR with KOD-plus Neo DNA polymerase (Toyobo) from plasmids including these Fab fragment genes [6] using primers Universal-OL1 and Myc-R or Universal-OL2 and FLAG-R, respectively. The PCR products were cloned into pCR2.1-TOPO vector (Invitrogen) and confirmed by an ABI PRISM 3100 genetic analyzer (Applied Biosystems). DNA templates of the $\mathrm{H}$ chain and $\mathrm{L}$ chain genes for mRNA display were prepared by PCR from these plasmids using primers Universal-OL1 and Myc25A-R, or Universal-OL2 and FLAG25A-R, respectively.

3.2. Library Construction. A randomized anti-p53 Fab fragment library was constructed by introducing point mutation to the wild type with the combination of error-prone PCR and StEP [24]. Mutazyme II DNA polymerase (Stratagene) was used for error-prone PCR and $15 \mathrm{ng}$ of either the $\mathrm{H}$ chain gene or the L chain gene constructed above was amplified for 30 cycles according to the protocol. After error-prone PCR, StEP was performed using Ex Taq DNA polymerase (Takara) as follows: denaturation at $95^{\circ} \mathrm{C}$ for $2 \mathrm{~min}, 80 \mathrm{cycles}$ of $95^{\circ} \mathrm{C}$ for $30 \mathrm{sec}$ and $55^{\circ} \mathrm{C}$ for $5 \mathrm{sec}$, followed by $95^{\circ} \mathrm{C}$ for $30 \mathrm{sec}$, $60^{\circ} \mathrm{C}$ for $30 \mathrm{sec}$, and $72^{\circ} \mathrm{C}$ for $15 \mathrm{~min}$. The PCR products were then resolved by agarose gel electrophoresis, extracted and purified with the QIAquick gel extraction kit (Qiagen). Error-prone PCR and StEP was performed once more each under the same condition. At every step, primers UniversalOL1 and Myc25A-R or Universal-OL2 and FLAG25A-R were used for the $\mathrm{H}$ chain and $\mathrm{L}$ chain genes, respectively.

3.3. In Vitro Transcription and Translation. The DNA templates were transcribed by T7 Ribomax Express Large Scale RNA production system (Promega) and purified by using the RNeasy mini kit (Qiagen). The transcribed mRNAs were then ligated with a puromycin linker by T4 RNA ligase (Takara) as described in previous studies [5]. Translation was done by PURE system S-S (PostGenome Institute) and 
TABLE 1: Oligonucleotide sequences.

\begin{tabular}{ll}
\hline Name & Sequence $\left(5^{\prime}\right.$ to $\left.3^{\prime}\right)$ \\
\hline Universal-OL1 & GAAATTAATACGACTCACTATAGGGAAACCACTACGGTCTCCCTCTAGAAATAATTTTGTTTAACTTTAAGAAG \\
Myc-R & GAGATATACCA \\
Universal-OL2 & GAAATTAATACGACTCACTATAGGGAGACCGTAGTGGTTTCCCTCTAGAAATAATTTTGTTTAACTTTAAGAAG \\
FLAG-R & GAGATATACCA \\
Myc25A-R & TTTTTTTTTTTTTTTTTTTTTTTTTTTAAGGTCTTCTTCACTAATCAGTTTCTGCTC \\
FLAG25A-R & TTTTTTTTTTTTTTTTTTTTTTTTTCTTGTCGTCATCGTCCTTGTAGTC \\
PolyT oligo & TTTTTTTTTTTTTTTTTTTTTTTTT \\
UnivOL1-F & GAAATTAATACGACTCACTATAGGGAAACCACTACGGTC \\
UnivOL2-F & GAAATTAATACGACTCACTATAGGGAGACCGTAGTGGTT \\
Fluo-F & CAGGATCGAGTGGGTCAAAC \\
Fluo-R & GGTGCTGGAGCTTTTGTCTG \\
p53-F & GGCAGAGCTTGTAAGGTCAG \\
p53-R & CAATCCATCCAATCCACTCC \\
\hline
\end{tabular}

The bold type resembles the sequences of the overlapping region for overlap-extension PCR.

Poly T oligo is labeled with a photo-cleavable biotin at the $5^{\prime}$ end.

5 pmol each of $\mathrm{H}$ and $\mathrm{L}$ chain templates were translated separately in a $25 \mu \mathrm{L}$ scale for 2 hours at $37^{\circ} \mathrm{C}$ to form mRNAdisplayed molecules. The molecules were then diluted into $175 \mu \mathrm{L}$ of Hybridization Buffer $(1 \mathrm{M} \mathrm{NaCl}, 100 \mathrm{mM}$ Tris$\mathrm{HCl}, \mathrm{pH} 7.4,10 \mathrm{mM}$ EDTA, and $0.25 \%$ Triton X-100) and mixed with $100 \mathrm{pmol}$ of poly-dT oligonucleotide with a biotinylated photo-cleavable linker and a high-capacity NeutrAvidin agarose resin (Pierce). The resin mixture was mixed gently at $4^{\circ} \mathrm{C}$ for 1 hour and subsequently washed with PBST (PBS with $0.1 \%$ Tween 20) three times. The resin was then mixed with ReverTra Ace reverse transcriptase (Toyobo) and incubated at $42^{\circ} \mathrm{C}$ for $30 \mathrm{~min}$. After washing with PBST three times, $40 \mu \mathrm{L}$ of elution buffer (PBS with $10 \%$ Solution A of PURE system S-S) was added to the resin and exposed to $\mathrm{UV}$ radiation at $>300 \mathrm{~nm}$ to elute the mRNA-displayed molecules as previously described [25]. The purified mRNAdisplayed $\mathrm{H}$ and $\mathrm{L}$ chains were mixed and then incubated overnight at $4^{\circ} \mathrm{C}$ to form mRNA-displayed Fab fragments.

3.4. Gene-linking Emulsion PCR. Emulsions were prepared by stirring $50 \mu \mathrm{L}$ of PCR reagents containing the selected mRNA-displayed Fab fragments, KOD-plus Neo DNA polymerase, and primers Myc-R and FLAG-R into $950 \mu \mathrm{L}$ of mineral oil-surfactant mixture [mineral-oil (Nacalai Tesque) containing $0.45 \%$ Span 85 (Nacalai Tesque), 0.04\% Tween 20 (Sigma), and $0.01 \%$ Triton X-100 (Nacalai Tesque)] at $2300 \mathrm{rpm}$ for $30 \mathrm{sec}$ at $4^{\circ} \mathrm{C}$. The emulsions were dispensed into $0.2 \mathrm{ml}$ PCR tubes $80-\mu \mathrm{L}$ each and PCR was performed with a T1 thermocycler (Biometra). The PCR program was as follows: denaturation at $94^{\circ} \mathrm{C}$ for $2 \mathrm{~min} ; 40-50$ cycles of $98^{\circ} \mathrm{C}$ for $10 \mathrm{sec}, 60^{\circ} \mathrm{C}$ for $30 \mathrm{sec}$, and $68^{\circ} \mathrm{C}$ for $1 \mathrm{~min}$; final extension at $68^{\circ} \mathrm{C}$ for $4 \mathrm{~min}$. A $70 \mu \mathrm{L}$ aliquot of the top layer was collected from each PCR tube, moved to a $1.7 \mathrm{~mL}$ tube, mixed with $100 \mu \mathrm{L}$ of $\mathrm{ddH}_{2} \mathrm{O}$, and centrifuged at $15,000 \mathrm{rpm}$ for $10 \mathrm{~min}$ at $40^{\circ} \mathrm{C}$ to break the emulsions. Approximately
$90 \%$ of the aqueous layer from the bottom was recovered and purified by briefly mixing it with $1 \mathrm{~mL}$ of mineral oil and centrifuging the mixture at $15,000 \mathrm{rpm}$ for $2 \mathrm{~min}$. From the purified aqueous layer, a $120 \mu \mathrm{L}$ aliquot was recovered and purified by using the QIAquick PCR purification kit (Qiagen). Subsequently, the PCR products were resolved by agarose gel electrophoresis, extracted, and purified with the QIAquick gel extraction kit.

3.5. Affinity Selection. Biotinylated antigen (either fluorescein (Sigma) or p53 C-terminal peptide (SKKGQSYSRH)) or biotin was added to $100 \mu \mathrm{L}$ of Magnotex-SA beads (Takara) dispersed in PBST, gently mixed at $4^{\circ} \mathrm{C}$ for 1 hour. After washing with PBST three times, $100 \mu \mathrm{L}$ of blocking buffer (DIG wash and block buffer set; Roche) was added and gently mixed at $4^{\circ} \mathrm{C}$ for another hour and finally washed with PBST to prepare antigen-immobilized beads or mock beads, respectively. To these antigen-immobilized beads or mock beads $5 \mu \mathrm{L}$ of sonicated salmon sperm DNA (Stratagene), $5 \mu \mathrm{L}$ of yeast tRNA (Invitrogen) and $40 \mu \mathrm{L}$ mRNA-displayed Fab fragments were added. After having been gently mixed for $1 \mathrm{hr}$ at $4^{\circ} \mathrm{C}$ for binding, the beads were washed with PBST three times and heated at $70^{\circ} \mathrm{C}$ for $15 \mathrm{~min}$ to elute the bound mRNA-displayed Fab fragments. Subsequently, emulsion PCR was performed as described above to link the corresponding $\mathrm{H}$ and $\mathrm{L}$ chain genes together.

To regenerate the genes, PCR was performed with KODplus Neo DNA polymerase using primers UnivOL1-F and Myc-R or UnivOL2-F and FLAG-R for the $\mathrm{H}$ chain and $\mathrm{L}$ chain genes, respectively. To prepare the DNA templates for the next round of selection, the regenerated $\mathrm{H}$ chain and L chain genes were amplified by StEP using Ex Taq DNA polymerase and the same primers and program described above with exception that the cycle numbers ranged from 60 80 cycles. The PCR products were then resolved by agarose 
gel electrophoresis, extracted and purified with the QIAquick gel extraction kit.

After several rounds of selection, the selected DNA was cloned using a TOPO XL PCR cloning kit (Invitrogen) and sequenced with the ABI PRISM 3100 genetic analyzer.

3.6. Quantitative PCR. The DNA amount of each gene was quantified by real-time PCR using SYBR premix Ex Taq DNA polymerase (Takara) and Lightcycler (Roche). Primers Fluo$\mathrm{F}$ and Fluo-R were used for anti-fluorescein Fab fragment genes, and primers p53-F and p53-R were used for anti-p53 Fab fragment genes.

3.7. ELISA. Fab fragments were expressed by transcribing and translating the $\mathrm{H}$ chain and $\mathrm{L}$ chain gene DNA with the PURE system S-S. Meanwhile, a p53 C-terminal peptideimmobilized plate was prepared by adding $100 \mathrm{pmol}$ of biotinylated p53 C-terminal peptide and $100 \mu \mathrm{L}$ of TBST (TBS with $0.1 \%$ Tween 20) to a Streptavidin C8 transparent plate (Nunc) and shaking it for 1 hour at room temperature. The plate was then washed with TBST 10 times and blocked with $200 \mu \mathrm{L}$ of blocking buffer by shaking it for 1 hour at room temperature. Separately, the expressed Fab fragments were diluted into $100 \mu \mathrm{L}$ of TBST containing $0-1000 \mathrm{nM} \mathrm{p} 53$ C-terminal peptide as a competitor and preincubated at $4^{\circ} \mathrm{C}$ for 1 hour. Then, the samples were added to the fluoresceinimmobilized plate and shaken for $5 \mathrm{~min}$. After a washing step, $100 \mu \mathrm{l}$ of TBST with $0.1 \%$ anti-FLAG M2 peroxidase conjugate (Sigma) was added and shaking was continued for 1 hour. The plate was washed for the last time and $100 \mu \mathrm{L}$ of TMB (Nacalai Tesque) was added. The plate was shaken for 20 min and then $100 \mu \mathrm{L}$ of $1 \mathrm{~N} \mathrm{H}_{2} \mathrm{SO}_{4}$ was added to stop the reaction. The absorbance at $450 \mathrm{~nm}$ was measured (reference wavelength: $655 \mathrm{~nm}$ ). The $K_{d}$ values of the selected clones were estimated from the Scatchard plot.

\section{Acknowledgments}

This work was supported by a Grant-in-Aid for JSPS Fellows (23-2677), a Grant-in-Aid for Scientific Research (19360377, 22360351), and a Strategic Research Foundation GrantAided Project for Private Universities (S0801008) from the MEXT of Japan. T. Sumida is supported by a scholarship from Keio-Kougakukai.

\section{References}

[1] H. R. Hoogenboom, "Selecting and screening recombinant antibody libraries," Nature Biotechnology, vol. 23, no. 9, pp. 1105-1116, 2005.

[2] H. R. Hoogenboom, A. D. Griffiths, K. S. Johnson, D. J. Chiswell, P. Hudson, and G. Winter, "Multi-subunit proteins on the surface of filamentous phage: methodologies for displaying antibody (Fab) heavy and light chains," Nucleic Acids Research, vol. 19, no. 15, pp. 4133-4137, 1991.

[3] V. Quintero-Hernández, V. R. Juárez-González, M. OrtízLeón, R. Sánchez, L. D. Possani, and B. Becerril, "The change of the scFv into the Fab format improves the stability and in vivo toxin neutralization capacity of recombinant antibodies," Molecular Immunology, vol. 44, no. 6, pp. 1307-1315, 2007.

[4] J. Hanes and A. Plückthun, "In vitro selection and evolution of functional proteins by using ribosome display," Proceedings of the National Academy of Sciences of the United States of America, vol. 94, no. 10, pp. 4937-4942, 1997.

[5] I. Fukuda, K. Kojoh, N. Tabata et al., "In vitro evolution of single-chain antibodies using mRNA display," Nucleic Acids Research, vol. 34, no. 19, article e127, 2006.

[6] T. Sumida, N. Doi, and H. Yanagawa, "Bicistronic DNA display for in vitro selection of Fab fragments," Nucleic Acids Research, vol. 37, no. 22, article e147, 2009.

[7] D. S. Tawfik and A. D. Griffiths, "Man-made cell-like compartments for molecular evolution," Nature Biotechnology, vol. 16, no. 7, pp. 652-656, 1998.

[8] M. Nakano, J. Komatsu, S. I. Matsuura, K. Takashima, S. Katsura, and A. Mizuno, "Single-molecule PCR using waterin-oil emulsion," Journal of Biotechnology, vol. 102, no. 2, pp. 117-124, 2003.

[9] T. Kojima, Y. Takei, M. Ohtsuka, Y. Kawarasaki, T. Yamane, and H. Nakano, "PCR amplification from single DNA molecules on magnetic beads in emulsion: application for high-throughput screening of transcription factor targets," Nucleic Acids Research, vol. 33, no. 17, article e150, 2005.

[10] R. Williams, S. G. Peisajovich, O. J. Miller, S. Magdassi, D. S. Tawfik, and A. D. Griffiths, "Amplification of complex gene libraries by emulsion PCR," Nature Methods, vol. 3, no. 7, pp. 545-550, 2006.

[11] F. Diehl, M. Li, Y. He, K. W. Kinzler, B. Vogelstein, and D. Dressman, "BEAMing: single-molecule PCR on microparticles in water-in-oil emulsions," Nature Methods, vol. 3, no. 7, pp. 551-559, 2006.

[12] L. Jermutus, A. Honegger, F. Schwesinger, J. Hanes, and A. Plückthun, "Tailoring in vitro evolution for protein affinity or stability," Proceedings of the National Academy of Sciences of the United States of America, vol. 98, no. 1, pp. 75-80, 2001.

[13] C. Zahnd, S. Spinelli, B. Luginbühl, P. Amstutz, C. Cambillau, and A. Plückthun, "Directed in Vitro evolution and crystallographic analysis of a peptide-binding single chain antibody fragment (scFv) with low picomolar affinity," Journal of Biological Chemistry, vol. 279, no. 18, pp. 18870-18877, 2004.

[14] B. Luginbühl, Z. Kanyo, R. M. Jones et al., "Directed evolution of an anti-prion protein scFv fragment to an affinity of $1 \mathrm{pM}$ and its structural interpretation," Journal of Molecular Biology, vol. 363, no. 1, pp. 75-97, 2006.

[15] G. Thom, A. C. Cockroft, A. G. Buchanan et al., "Probing a protein-protein interaction by in vitro evolution," Proceedings of the National Academy of Sciences of the United States of America, vol. 103, no. 20, pp. 7619-7624, 2006.

[16] H. Persson, H. Wallmark, A. Ljungars, J. Hallborn, and M. Ohlin, "In vitro evolution of an antibody fragment population to find high-affinity hapten binders," Protein Engineering, Design and Selection, vol. 21, no. 8, pp. 485-493, 2008.

[17] Y. Shimizu, T. Kanamori, and T. Ueda, "Protein synthesis by pure translation systems," Methods, vol. 36, no. 3, pp. 299-304, 2005.

[18] P. A. C. 'T Hoen, S. M. G. Jirka, B. R. Ten Broeke et al., "Phage display screening without repetitious selection rounds," Analytical Biochemistry, vol. 421, no. 2, pp. 622-631, 2012.

[19] N. Tabata, Y. Sakuma, Y. Honda et al., "Rapid antibody selection by mRNA display on a microfluidic chip," Nucleic Acids Research, vol. 37, no. 8, article e64, 2009. 
[20] S. R. Nirantar and F. J. Ghadessy, "Compartmentalized linkage of genes encoding interacting protein pairs," Proteomics, vol. 11, no. 7, pp. 1335-1339, 2011.

[21] C. Tuerk and L. Gold, "Systemic evolution of ligands by exponential enrichment: RNA ligands to bacteriophage T4 DNA polymerase," Science, vol. 249, no. 4968, pp. 505-510, 1990.

[22] T. Morii, M. Hagihara, S. I. Sato, and K. Makino, "In vitro selection of ATP-binding receptors using a ribonucleopeptide complex," Journal of the American Chemical Society, vol. 124, no. 17, pp. 4617-4622, 2002.

[23] S. I. Sato, M. Fukuda, M. Hagihara, Y. Tanabe, K. Ohkubo, and T. Morii, "Stepwise molding of a highly selective ribonucleopeptide receptor," Journal of the American Chemical Society, vol. 127, no. 1, pp. 30-31, 2005.

[24] H. Zhao and W. Zha, "In vitro 'sexual' evolution through the PCR-based staggered extension process (StEP)," Nature Protocols, vol. 1, no. 4, pp. 1865-1871, 2006.

[25] J. Olejnik, S. Sonar, E. Krzymañska-Olejnik, and K. J. Rothschild, "Photocleavable biotin derivatives: a versatile approach for the isolation of biomolecules," Proceedings of the National Academy of Sciences of the United States of America, vol. 92, no. 16, pp. 7590-7594, 1995. 

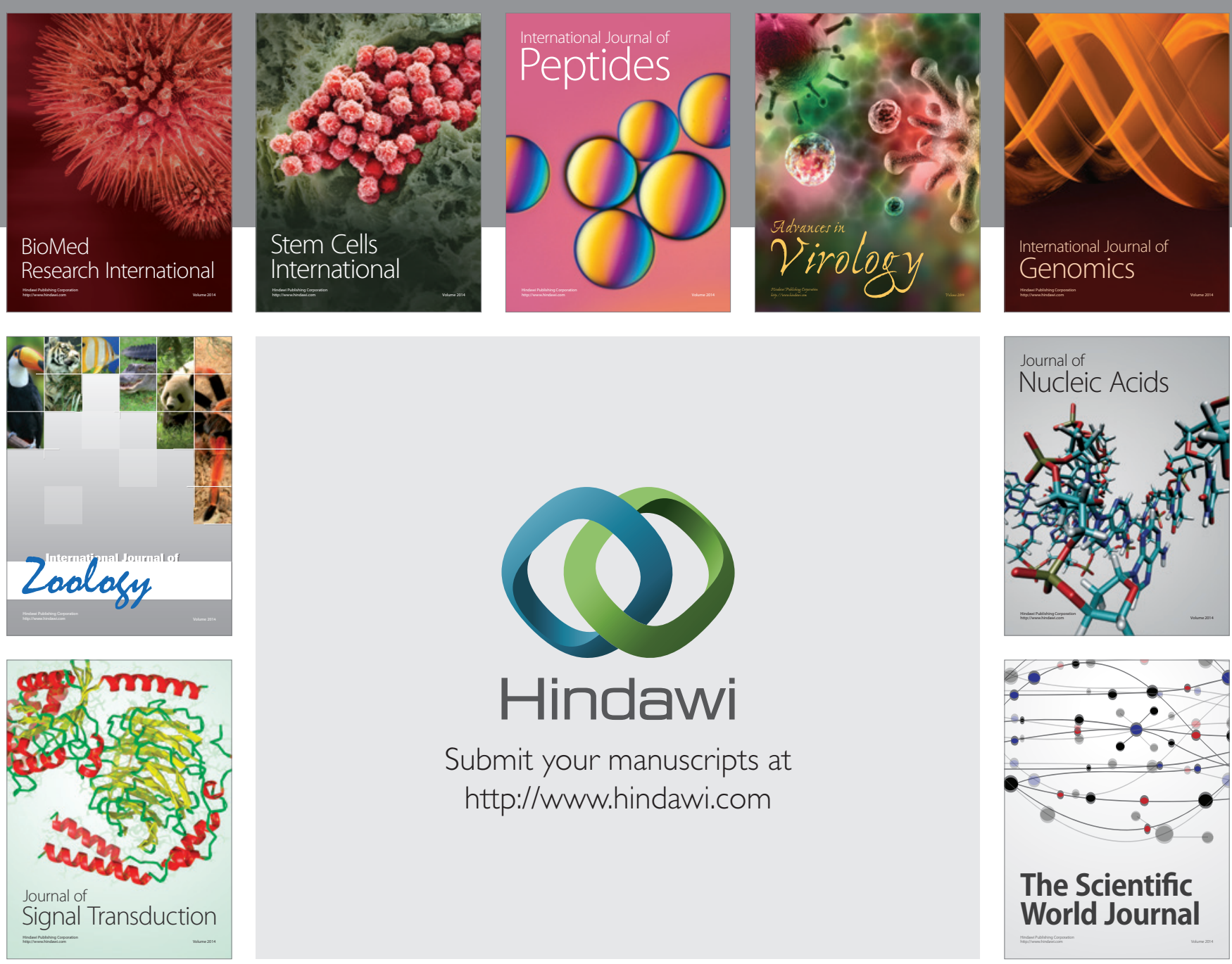

Submit your manuscripts at

http://www.hindawi.com
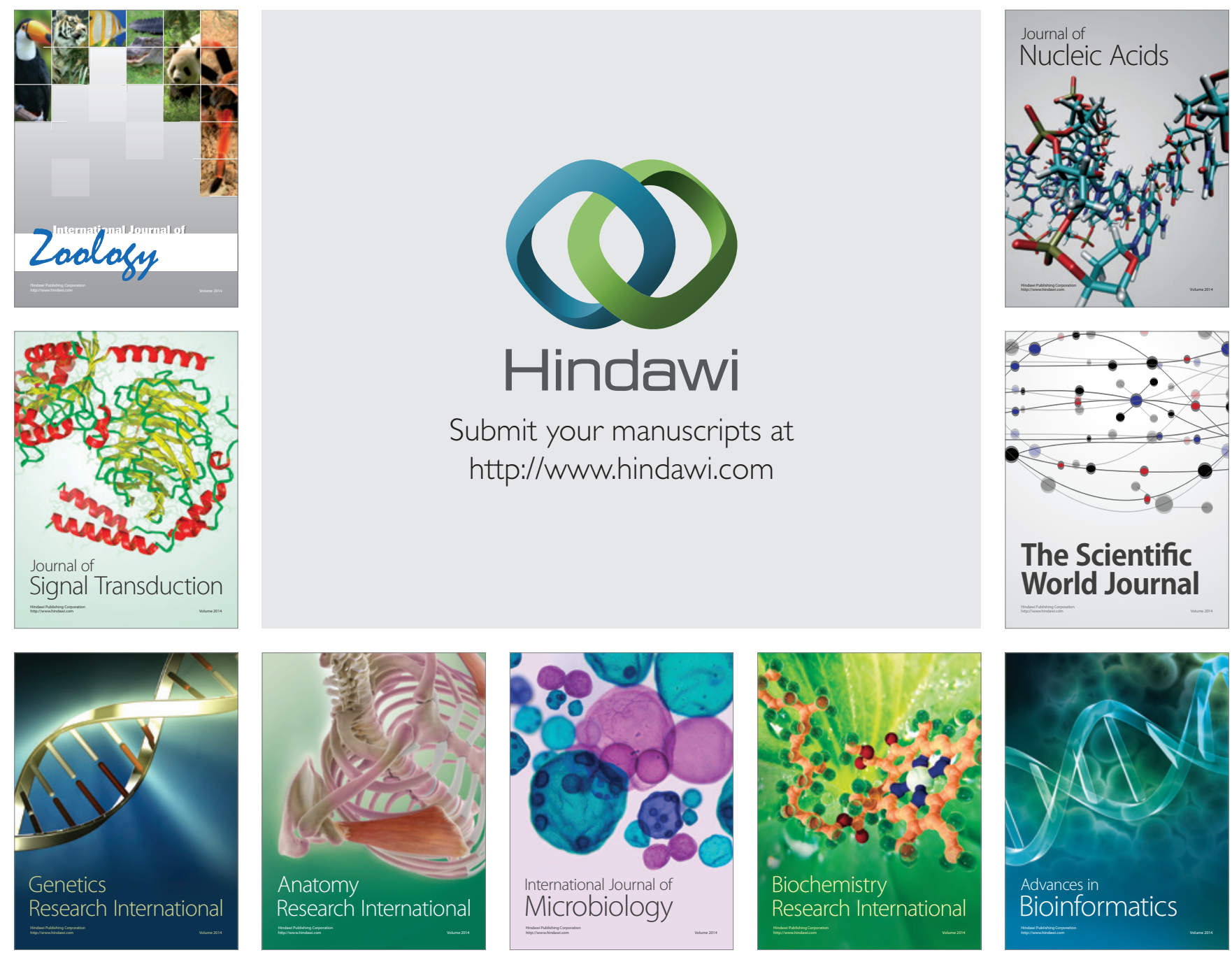

The Scientific World Journal
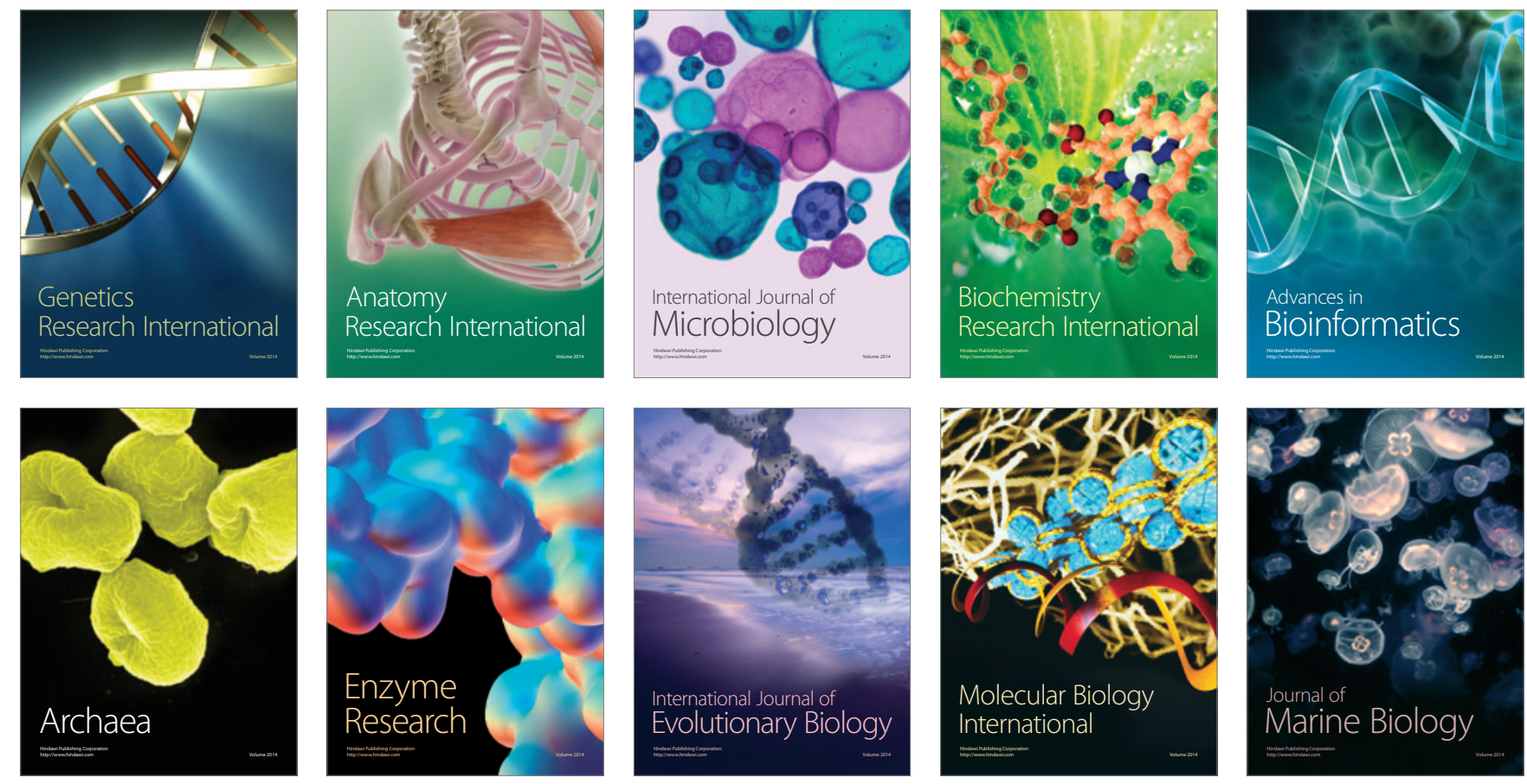